\title{
Capture Gamma-Ray Libraries for Nuclear Applications*
}

\author{
B.W. SLEAFORD ${ }^{*}, 1$, R.B. FIRESTONE ${ }^{2}$, N. SUMMERS ${ }^{1}$, J. ESCHER ${ }^{1}$, A. HURST ${ }^{1,2}$, M. KRTICKA ${ }^{3}$, S. BASUNIA ${ }^{2}$, G. $^{2}$ \\ MOLNAR $^{4}$, T. BELGYA ${ }^{4}$, Z. REVAY ${ }^{4}$, AND H.D. CHOI $^{5}$ \\ ${ }^{1}$ Lawrence Livermore National Laboratory, Livermore, California 94551 \\ ${ }^{2}$ Lawrence Berkeley National Laboratory, Berkeley, CA 94720 \\ ${ }^{3}$ Charles University in Prague, Faculty of Mathematics and Physics, V Holešovičkách 2, CZ-180 00 Prague 8, Czech Republic \\ ${ }^{4}$ Institute of Isotope and Surface Chemistry, H-1525, Budapest, Hungary \\ ${ }^{5}$ Seoul National University, Republic of Korea \\ ${ }^{*}$ Corresponding author. E-mail : sleaford1@1lnl.gov \\ Received \\ Accepted for Publication
}

The neutron capture reaction is useful in identifying and analyzing the gamma-ray spectrum from an unknown assembly as it gives unambiguous information on its composition. This can be done passively or actively where an external neutron source is used to probe an unknown assembly. There are known capture gamma-ray data gaps in the ENDF libraries used by transport codes for various nuclear applications. The Evaluated Gamma-ray Activation file (EGAF) is a new thermal neutron capture database of discrete line spectra and cross sections for over 260 isotopes that was developed as part of an IAEA Coordinated Research Project. EGAF has been used to improve the capture gamma production in ENDF libraries. For medium to heavy nuclei the quasi continuum contribution to the gamma cascades is not experimentally resolved. The continuum contains up to $90 \%$ of all the decay energy an is modeled here with the statistical nuclear structure code DICEBOX. This code also provides a consistency check of the level scheme nuclear structure evaluation. The calculated continuum is of sufficient accuracy to include in the ENDF libraries. This analysis also determines new total thermal capture cross sections and provides an improved RIPL database. For higher energy neutron capture there is less experimental data available making benchmarking of the modeling codes more difficult. We use CASINO, a version of DICEBOX that is modified for this purpose. This can be used to simulate the neutron capture at incident neutron energies up to $20 \mathrm{MeV}$ to improve the gamma-ray spectrum in neutron data libraries used for transport modelling of unknown assemblies.

KEYWORDS : ND2010, Nuclear Data, ENDF

\section{INTRODUCTION}

Prompt gamma-rays emitted following neutron capture provide unique elemental signatures that provide unambiguous information on the composition of unknown assemblies. For many years no reliable prompt gamma-ray database existed for this kind of analysis. Recently new, precise, prompt gamma-ray measurements have been made with the guided thermal neutron beam at the Budapest Reactor on all elemental targets with $Z=1-83$, 90, 92 with the exception of helium and promethium. These data have been evaluated and published as part of an IAEA Coordinated Research Project [1].

For light isotopes the neutron capture decay scheme is nearly complete, but for medium and heavy isotopes considerable unresolved continuum gamma-ray feeding exists. This feeding can be accounted for using the statistical model code DICEBOX [2]. These calculations can also be used to determine total radiative thermal neutron capture cross sections $\sigma_{0}$, capture state spin distributions, and improved nuclear structure information from the EGAF data.

Relatively little experimental data are available for prompt gamma-ray emission following higher energy neutron capture. These data can be accurately calculated with the CASINO version of DICEBOX [3]. We are evaluating the EGAF data using the DICEBOX and CASINO codes to develop a comprehensive ENDF capture gamma-ray library for use in transport modelling of unkown assemblies.

\footnotetext{
${ }^{1}$ Present address: Department of Physics, University of Hankook, Seoul, 120-424, Korea
} 


\subsection{Experimental Measurements}

Neutron capture gamma-ray cross sections were measured with the guided neutron beam at the 10-MW Budapest Reactor [4]. The target station is located $\approx 30 \mathrm{~m}$ from the reactor where both primary and secondary gamma-rays can be measured in low background conditions. Neutrons enter the evacuated target holder and continue to the beam stop at the rear wall of the guide hall. The thermal-equivalent neutron flux was $2 \times 10^{6} \mathrm{n} \mathrm{cm}^{2} \mathrm{~s}^{-1}$.

Prompt gamma-rays from the target were measured with an n-type high-purity, 25\% efficient (HPGe) detector with closed-end coaxial geometry located $23.5 \mathrm{~cm}$ from the target. The detector is Compton-suppressed by a BGO-scintillator guard detector annulus surrounded by $10-\mathrm{cm}$ thick lead shielding. Counting efficiency was calibrated from $50 \mathrm{keV}$ to $10 \mathrm{MeV}$ with radioactive sources and $(n, \gamma)$ reaction gamma rays to a precision of better that $1 \%$ from $500 \mathrm{keV}$ to $6 \mathrm{MeV}$ and better than $3 \%$ at all other energies [5]. The gamma-ray spectra were analyzed using the Hypermet PC program [5,6].

Elemental radiative thermal neutron gamma-ray cross sections were calibrated using natural elemental targets of stoichiometric compounds or homogenous mixtures containing elements with well known cross sections e.g. H, $\mathrm{N}, \mathrm{Cl}, \mathrm{S}, \mathrm{Na}$, Ti, or Au [7]. The guided neutron beam used in these measurements is a purely thermal so no correction was necessary for epithermal contributions to the measured gamma-ray cross sections. The gamma-rays were assigned to the isotopic level scheme on the basis of energy and intensity by comparison with data from the ENSDF file [8]. Elemental gamma-ray cross sections were measured and isotopic cross sections determined assuming the normal abundances [9].

\subsection{EGAF Database}

Until recently there was no reliable neutron capture gamma-ray database although these data are also an important component of the Evaluated Neutron Data File (ENDF) used in neutron transport calculations. Groshev et al [10] published the first compilation of prompt capture $\gamma$-ray energies and intensities, and Greenwood et al [11] the first spectrum catalog during the era of $\mathrm{NaI}$ detectors. With the advent of Ge detectors in the 1960s, Rasmussen [12] and Orphan [13] measured capture gamma-ray spectra for all elements. These data were compiled by Lone et al [14] who published a database of over 10,000 gamma-rays in 1981 . This database was used for many years despite the inadequacies inherent to those early measurements. Prompt neutron capture $\gamma$-ray data are also compiled from the literature in the Evaluated Nuclear Structure Data File (ENSDF) [8]. These data were used primarily to extract nuclear structure information and were not evaluated for applied use.

Reedy and Frankel [15] carefully reevaluated the literature for light elements from hydrogen to zinc and provided this information in ENSDF format. The Lone et al, ENSDF, and Reedy gamma-ray intensities are normalized to units of per 100 neutron captures. In the 1990's new capture gamma-ray measurements were performed for all stable elements by Molnar et al at the Budapest Reactor. An International Atomic Energy Agency (IAEA) Coordinated Research Project (CRP) was organized to evaluate these data. The IAEA CRP produced a database of $\approx 35,000$ neutron capture $\gamma$-rays [16] which was named the Evaluated Gamma-ray Activation File (EGAF).

\section{STATISTICAL MODEL CALCULATIONS}

Theoretical feedings of low-lying levels by thermal neutron radiative capture were calculated using the computer code DICEBOX [2]. The algorithm of this Monte Carlo code is based on the generalization of the extreme statistical model embodying Bohr's idea of a compound nucleus [17]. Below a certain critical energy, $E_{\text {crit }}$, the level scheme, i.e. energies, spins and parities of all levels as well as all de-exciting transitions, is taken from experiment. Above $\mathrm{E}_{\text {crit }}$, a set of levels is generated as a random discretization of an a priori known level density formula $\rho\left(E, J^{\pi}\right)$. Decay properties of an initial level $i$ above $\mathrm{E}_{\text {crit }}$ are completely characterized by a full set of partial radiation widths to all final levels $f$ below the level $i$. A partial radiative width, $\Gamma_{\mathrm{i} \gamma \mathrm{f}}$, which characterizes the probability of gamma-ray decay with an energy $E_{\gamma}=E_{i}-E_{f}$ is assumed to be a random choice from the Porter-Thomas distribution [18] with a mean value

$$
\left\langle\Gamma_{i \not f}\right\rangle=\frac{f^{(X L)}\left(E_{\gamma}, \xi\right) \times E_{\gamma}^{3}}{\rho\left(E_{i}, J_{i}^{\pi}\right)}
$$

Here $\rho\left(E_{i}, J_{i}^{\pi}\right)$ is the level density near the initial level $i$ and $f^{(X L)}\left(E_{\gamma}, \xi\right)$ is the photon strength function (PSF) for a transition of given type $X$ and multipolarity $L$. The argument $\xi$ of the PSF represents possible dependence on quantities other than gamma-ray energy. In the extreme statistical model it is assumed that the $\Gamma_{i \gamma f}$ are uncorrelated. Selection rules for different types of transitions are fully accounted for in the generation of $\Gamma_{i \gamma \text {. }}$.

The random generation of a system of all $\Gamma_{i y f}$, which fully describe the decay properties of the nuclear levels is called a nuclear realization. Due to fluctuations involved there exists an almost infinite number of nuclear realizations, that differ in decay properties even for a single choice of $f^{(X L)}$ and level density. Consequently all simulated quantities are subject to statistical fluctuations arising from different nuclear realizations. Determination of these fluctuations with the DICEBOX code allows us to estimate the uncertainty coming from statistical nature of decay process. 
Typically a calculation consists of 50 nuclear realizations, each with 50000 capture state decays, generated by the Monte Carlo method. DICEBOX stores the simulated capture state deexcitation data which are used to calculate populations of low-lying levels below $\mathrm{E}_{\text {crit }}$ and intensities of all gamma rays per neutron capture. Simulated quantities can be renormalized to absolute cross sections by comparison with the experimental gamma-ray cross sections depopulating low-lying levels.

Energy dependence and absolute values of $f^{(X L)}\left(E_{\gamma}, \xi\right)$ are not sufficiently known for nuclei with $\mathrm{A}<50$. We therefore have used only simple models for these quantities in our simulations. For E1 transitions we adopted three different models: (i) The Lorentzian shape of Giant Dipole Electric Resonance (GDER) [19] in conjunction with the Brink hypothesis [20], also called the Standard Lorentzian (SLO) model, (ii) the KMF model [21], which modifies the shape of low-energy tail of GDER, and (iii) the single-particle (SP) model [22] where $f^{(E 1)}=$ const. is independent of gamma-ray energy. Parameters of GDER can be found in the survey of Dietrich and Berman [23].

For $M 1$ strength two different models were used: (i) the single-particle SP model where $f^{(M 1)}=$ const, and (ii) a model where $f^{(M I)}$ is described as Lorentzian-shaped resonance Giant Dipole Magnetic resonance (GDMR) at about $12 \mathrm{MeV}$ with a width about $3 \mathrm{MeV}$ [24]. The strengths of $f^{(M 1)}$ were adjusted to match the observed population of positive and negative parity levels at low excitation energies. For E2 strength a single-particle SP model was used.

The Back-Shifted Fermi Gas (BSFG) and Constant-Temperature (CT) models, in the parametrization from von Egidy, Schmidt, and Bekhami [25], were used for level density. All possible combinations of $f^{(X L)}$ and level density were tested in these simulations.

\section{RESULTS}

\subsection{Low-Z Isotope Analysis}

For low-Z isotopes the EGAF prompt gamma-ray data are usually complete. For example the ${ }^{12} \mathrm{C}(\mathrm{n}, \gamma){ }^{13} \mathrm{C}$ decay scheme is shown in Fig. 1. All six gamma-rays from neutron capture are observed and the cross section balance through the decay scheme is excellent with 3.86(6) mb deexciting the capture state and $3.85(6) \mathrm{mb}$ populating the ground state. This value is significantly larger than 3.53(7) $\mathrm{mb}$ in ENDF, adopted by a measurement by Jurney et al [26], but consistent with other values given in Table 1. The Jurney et al measurement of the ${ }^{2} \mathrm{H}$ total radiative thermal neutron cross section, $\sigma_{0}=0.508(15) \mathrm{mb}$, was also measured in [26] using the lower ${ }^{12} \mathrm{C}$ cross section as a standard. As shown in Table 1 renormalizing this cross section gives a new value, $\sigma_{0}=0.552(16) \mathrm{mb}$, also consistent with other values.

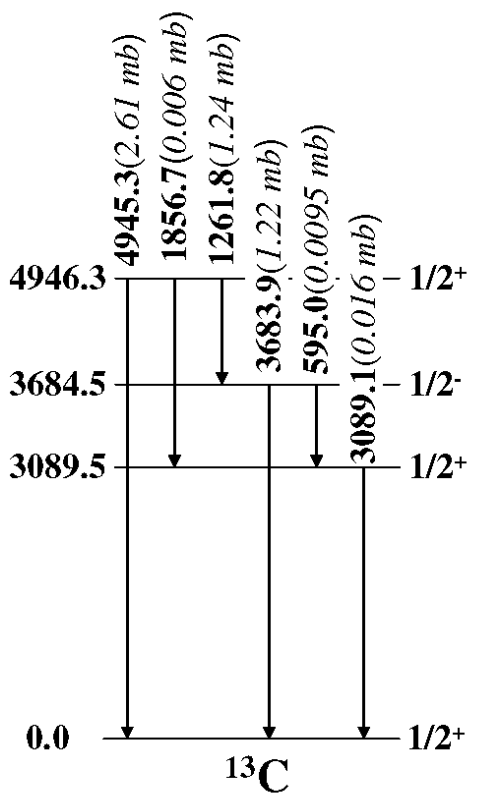

Figure $1 .{ }^{12} \mathrm{C}(\mathrm{n}, \gamma){ }^{13} \mathrm{C}$ thermal neutron capture decay scheme.

Table 1. Comparison previous measurements of ${ }^{2} \mathrm{H}$ and ${ }^{12} \mathrm{C}$ total radiative thermal neutron cross sections with EGAF results.

\begin{tabular}{|c|c|c|c|}
\hline Author (Year) & $\sigma 0(\mathbf{m b})$ & Author (Year) & $\sigma 0(\mathrm{mb})$ \\
\hline Prestwich(1981) & $3.50 \pm 0.16$ & Trail (1964) & $0.36 \pm 0.03$ \\
\hline Jurney (1982) & $3.53 \pm 0.07$ & Alfimenkov(1980) & $0.476 \pm 0.020$ \\
\hline Nichols (1960) & $3.57 \pm 0.03$ & Jurney (1982) & $0.508 \pm 0.015$ \\
\hline Sagot (1963) & $3.72 \pm 0.15$ & Merritt (1968) & $0.521 \pm 0.009$ \\
\hline Jurney (1963) & $3.8 \pm 0.4$ & Silk (1969) & $0.523 \pm 0.029$ \\
\hline Starr (1962) & $3.83 \pm 0.06$ & Ishikawa(1973) & $0.55 \pm 0.01$ \\
\hline Hennig (1967) & $3.85 \pm 0.15$ & Kaplan (1952) & $0.57 \pm 0.01$ \\
\hline Matsue (2004) & $3.81 \pm 0.11$ & Jurney (1963) & $0.60 \pm 0.05$ \\
\hline EGAF (2007) & $3.90 \pm 0.06$ & Sargent (1947) & $0.92 \pm 0.22$ \\
\hline ENDF & $3.53 \pm 0.07$ & ENDF & $0.508 \pm 0.015$ \\
\hline
\end{tabular}

\subsection{High-Z Isotope Analysis}

For high- $Z$ isotopes the neutron capture gamma-ray decay scheme is usually too complex to resolve all transitions. This is because as the level density becomes large level separations become smaller than the intrinsic resolution of HPGe detectors resulting in a high density, unresolved continuum of transitions. Often a large fraction of the neutron capture cross section will still populate the ground 
state through secondary transitions. Observed primary transitions may be much weaker, especially if the neutron separation energy $S_{n}$ is large, in which case most of the primary transitions will populate levels near $S_{n}$ where the level density is very high. An example is ${ }^{105} \mathrm{Pd}(\mathrm{n}, \gamma){ }^{106} \mathrm{Pd}$ where the neutron capture decay cross section balance is shown in Table 2. More than $20 \mathrm{~b}$ are observed feeding the ground state of ${ }^{106} \mathrm{Pd}$, nearly the $21.0(15) \mathrm{b}$ cross section recommended by Mughabghab [27]. By comparison only $0.55 \mathrm{~b}$ are observed in primary transitions deexciting the 9561-kev capture state.

Table 2. Cross section balance for ${ }^{105} \mathrm{Pd}(\mathrm{n}, \gamma){ }^{106} \mathrm{Pd}$

\begin{tabular}{|c|c|c|c|c|}
\hline $\mathrm{E}$ (level) & $\mathbf{J}^{\pi}$ & $\Sigma \sigma_{\gamma}($ in) & $\Sigma \sigma_{\gamma}$ (out) & $\Delta \Sigma \sigma$ \\
\hline 0 & $0+$ & 20.26 & & \\
\hline 511.844 & $2+$ & 13.88 & 17.91 & 4.03 \\
\hline 1128.04 & $2+$ & 2.371 & 4.263 & 1.892 \\
\hline 1133.79 & $0_{+}$ & 0.227 & 0.565 & 0.338 \\
\hline 1229.2 & $4+$ & 1.630 & 3.479 & 1.849 \\
\hline 1557.67 & $3+$ & 1.183 & 2.142 & 0.959 \\
\hline 1562.16 & $2+$ & 0.312 & 1.869 & 1.557 \\
\hline 1706.44 & $0_{+}$ & 0.012 & 0.193 & 0.181 \\
\hline 1909.39 & $2+$ & 0.063 & 0.724 & 0.661 \\
\hline 1932.37 & $4+$ & 0.217 & 0.590 & 0.373 \\
\hline 2001.56 & $0+$ & 0.029 & 0.118 & 0.089 \\
\hline 2077.1 & $6+$ & 0.001 & 0.103 & 0.102 \\
\hline 2077.37 & $(4)+$ & 0.057 & 0.440 & 0.383 \\
\hline 2084.39 & -3 & 0.123 & 1.033 & 0.910 \\
\hline 2242.4 & $2+$ & 0.026 & 0.499 & 0.473 \\
\hline 2278.47 & $0+$ & 0 & 0.056 & 0.056 \\
\hline 2282.89 & $4+$ & 0.0007 & 0.275 & 0.274 \\
\hline 2306.01 & -3 & 0.053 & 0.542 & 0.489 \\
\hline 2308.73 & $2+$ & 0.000 & 0.283 & 0.283 \\
\hline 2350.96 & $4+$ & 0.018 & 0.304 & 0.286 \\
\hline 2366.09 & $5+$ & 0.003 & 0.116 & 0.114 \\
\hline 2397.37 & (5)- & 0.055 & 0.263 & 0.209 \\
\hline 2401 & $(2-, 3-)$ & 0.037 & 0.300 & 0.263 \\
\hline 2439.11 & $2+$ & 0.065 & 0.293 & 0.227 \\
\hline 2472.09 & $0+$ & 0.000 & 0.055 & 0.055 \\
\hline 2484.76 & $(1-)$ & 0.043 & 0.253 & 0.211 \\
\hline 2500.01 & -2 & 0.028 & 0.296 & 0.267 \\
\hline 2578.64 & (4-) & 0.00004 & 0.221 & 0.221 \\
\hline$\cdots$ & $\cdots$ & $\cdots$ & $\cdots$ & $\ldots$ \\
\hline $\begin{array}{c}\ldots \\
9561.4\end{array}$ & $\begin{array}{c}\cdots \\
2+, 3+\end{array}$ & $\cdots$ & $\begin{array}{c}\cdots \\
0.554\end{array}$ & $\cdots$ \\
\hline
\end{tabular}

Fortunately, we can calculate the missing continuum intensity with the statistical model code DICEBOX assuming only average properties for the continuum of transitions. Level density and photon strength parameters can be determined by comparisons with photonuclear data and experimental capture state widths. The qualtity of the analysis can be determined by comparing the observed transition cross section deexciting levels below $\mathrm{E}_{\text {crit }}$ with the calculated cross section populating these levels as shown in Fig.2. This fit can be optimized to determine the admixture of spins in the neutron capture state.

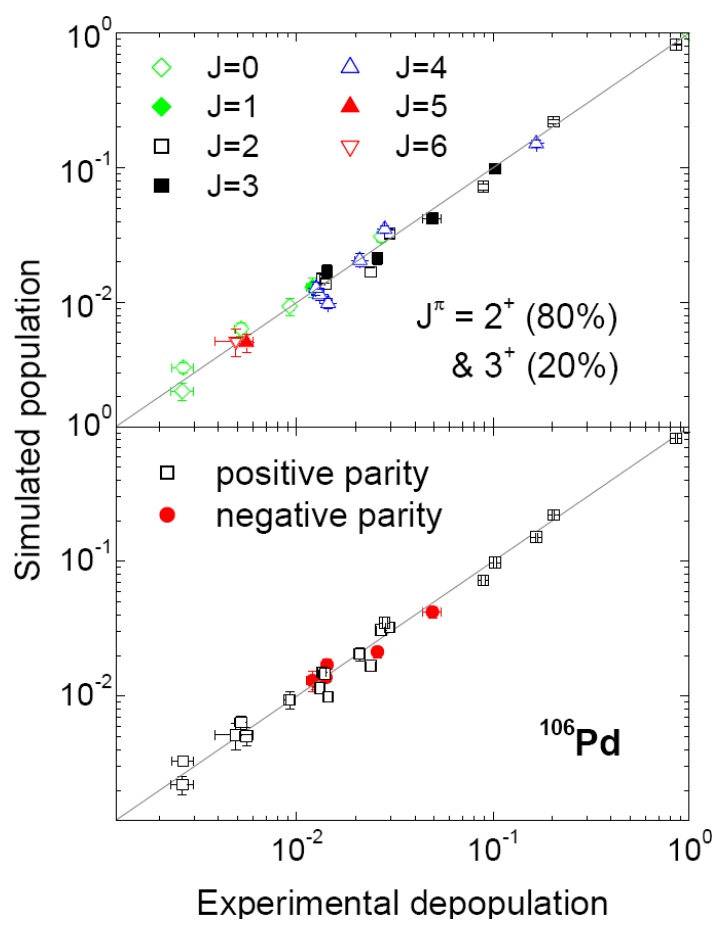

Figure 2. Population/depopulation plot for ${ }^{105} \mathrm{Pd}(\mathrm{n}, \gamma){ }^{106} \mathrm{Pd}$.

This statistical analysis can also be used to improve the nuclear structure data be low $\mathrm{E}_{\text {crit. }}$ When a poor fit is observed to a level in the population/depopulation plot it is likely that there is a problem with the $\mathrm{J}^{\pi}$ of that level. An example was found in ${ }^{184} \mathrm{~W}(\mathrm{n}, \gamma){ }^{185} \mathrm{~W}$, Fig. 3, where the 332-keV level was found to be more consistent with $9 / 2^{-}$ than $\left(7 / 2^{-}\right)$recommended in ENSDF [8]. In addition, nine new transitions were placed in the ${ }^{185} \mathrm{~W}$ level scheme from the EGAF data.

\section{CONCLUSIONS}

The use of EGAF data combined with statistical model calculations using DICEBOX provide a powerful method for improving the capture gamma-ray data in ENDF. A comparison of the original ENDF library data for ${ }^{104} \mathrm{Pd}(\mathrm{n}, \gamma){ }^{105} \mathrm{Pd}$ with out new, EGAF based library is shown in Fig. 4. Although the broad resolution of the original ENDF library is similar to the new library, narrow line information in the new library make this far more useful for the analysis of unknown assemblies. Table 3 privides a summary of the completed libraries to date. 
C. KIM et al., ND2010 Proceedings - Instructions for Authors

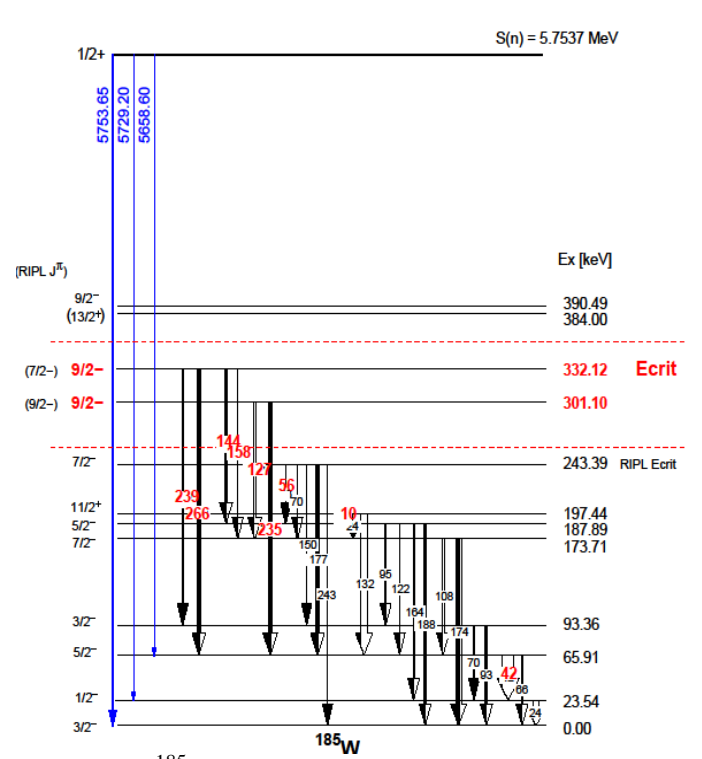

Figure 3. ${ }^{185} \mathrm{~W}$ level scheme determined using EGAF data with DICEBOX statistical model calculations. Two new levels and nine new transitions (red) were determined.

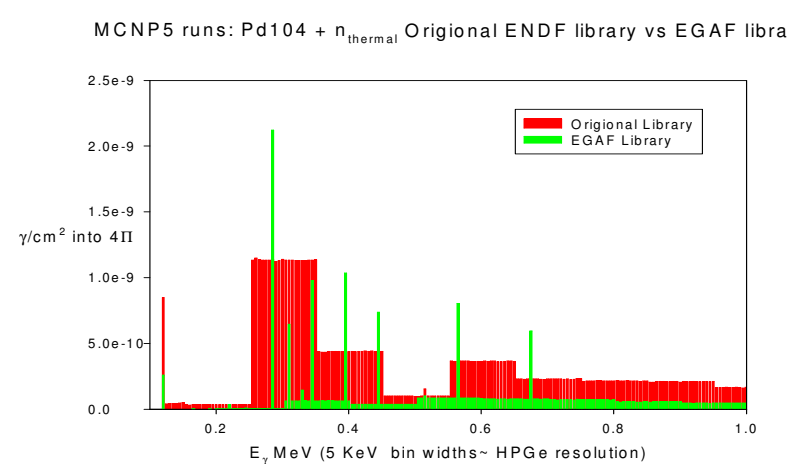

Figure 4. Comparison of the original ${ }^{104} \mathrm{Pd}(\mathrm{n}, \gamma){ }^{105} \mathrm{Pd}$ ENDF library with the new EGAF-based library.

\section{ACKNOWLEDGEMENTS}

This work was performed under the auspices of the U.S Department of Energy by the Unniversity of California, supported by the Director, Office of Science, Office of Basic Energy Sciences, of the U.S. Department of Energy at Lawrence Berkeley National Laboratory under Contract No. DE-AC02-05CH11231 and at Lawrence Livermore National Laboratory under Contract W-7505-Eng-48. Support was also provided by National Nuclear Security Administration Academic Alliance Grant No. DE-FG52-06NA26194 and by the research plan MSM 002 1620859 supplied by the Ministry of Education of the Czech Republic.
Table 3. EGAF capture gamma-ray library completion status.

\begin{tabular}{|c|c|c|c|c|c|}
\hline $\mathrm{Z}$ & El & A & $\% \mathrm{NA}$ & barns & $\mathrm{N} \gamma$ \\
\hline 1 & $\mathrm{H}$ & 1 & 99.9844 & 0.33260 & 1 \\
\hline 1 & $\mathrm{D}$ & 2 & 0.01557 & 0.00052 & 1 \\
\hline 3 & $\mathrm{Li}$ & 6 & 7.589 & 0.04000 & 3 \\
\hline 3 & $\mathrm{Li}$ & 7 & 92.411 & 0.05000 & 3 \\
\hline 4 & $\mathrm{Be}$ & 9 & 100 & 0.01000 & 12 \\
\hline 5 & B & 10 & 19.82 & 0.50000 & 9 \\
\hline 5 & B & 11 & 80.18 & 0.01000 & 9 \\
\hline 6 & $\mathrm{C}$ & 12 & 98.892 & 0.00353 & 6 \\
\hline 7 & $\mathrm{~N}$ & 14 & 99.6337 & 0.08000 & 60 \\
\hline 8 & $\mathrm{O}$ & 16 & 99.7628 & 0.00019 & 4 \\
\hline 9 & $\mathrm{~F}$ & 19 & 100 & 0.00960 & 1622 \\
\hline 11 & $\mathrm{Na}$ & 23 & 100 & 0.53000 & 233 \\
\hline 12 & $\mathrm{Mg}$ & 24 & & 0.06000 & 283 \\
\hline 13 & $\mathrm{Al}$ & 27 & 100 & 0.23000 & 291 \\
\hline 14 & $\mathrm{Si}$ & 28 & 92.2297 & 0.18000 & 54 \\
\hline 15 & $\mathrm{P}$ & 31 & 100 & 0.17000 & 202 \\
\hline 16 & $\mathrm{~S}$ & 32 & & 0.53000 & 470 \\
\hline 17 & $\mathrm{Cl}$ & 35 & 75.771 & 45.55000 & 383 \\
\hline 17 & $\mathrm{Cl}$ & 37 & 24.229 & 0.43000 & 77 \\
\hline 26 & $\mathrm{Fe}$ & 56 & 91.75 & 2.59000 & 193 \\
\hline 46 & $\mathrm{Pd}$ & 104 & 11.14 & 0.60000 & 13 \\
\hline 74 & $\mathrm{~W}$ & 182 & 26.4985 & 19.90000 & 126 \\
\hline 74 & W & 183 & 14.3136 & 10.30000 & 212 \\
\hline 74 & W & 184 & 30.6422 & 1.70000 & 64 \\
\hline 74 & W & 186 & 28.4259 & 38.50000 & 152 \\
\hline 82 & $\mathrm{~Pb}$ & 207 & 22.0827 & 0.62500 & 25 \\
\hline
\end{tabular}

\section{REFERENCES}

[1] Database of Prompt Gamma Rays from Slow Neutron Capture for Elemental Analysis, R.B. Firestone, et al, IAEA STI/PUB/1263, 251 pp (2007);

[2] F. Be`cvárr, Nucl. Instrum. Methods A 417, 434 (1998).

[3] F. Be`cvár, Statistical $\gamma$ Cascades Following Thermal and keV Neutron Capture in Heavy Nuclei. in S. Wender, ed., Gamma-Ray Spectroscopy and Related Topics. American Institute of Physics, New York (2000), 504.

[4] T. Belgya, Zs. RI' $\{$ e $\}$ vay, B. Fazekas, I. $\mathrm{H}^{\prime}\{\mathrm{e}\} \mathrm{jja}$, L. Dabolczi, G.L. Molnl'\{a\}r, Z. Kis, J. |" $\{\mathrm{O}\}$ stl" $\{\mathrm{o}\} \mathrm{r}$, Gy. Kaszl' $\{a\} s$ in Proc. 9th International Symposium on Capture Gamma-Ray Spectroscopy and Related Topics, Budapest, Hungary, Oct. 8-12, Eds. G. Molnl' $\{a\} r$, T. Belgya, Zs. Rl' $\{$ e $\}$ vay, Springer Verlag Budapest, Berlin, Heidelberg 1997 p. 826.

[5] G.L. Molnar, Zs. Revay, and T. Belgya, Nucl. Instrum. Meth. Phys. Res. A489, 140 (2002).

[6] B. Fazekas, J. $\sim "\{\mathrm{O}\}$ stl" $\{\mathrm{o}\} \mathrm{r}$, Z. Kis, G.L. Molnl' $\{\mathrm{a}\} \mathrm{r}$, A. $\sim$ Simonits in Proc. 9th International Symposium on Capture Gamma-Ray Spectroscopy and Related Topics, Budapest, Hungary, Oct. 8-12, Eds. G. Molnl' $\{a\}$ r, T. Belgya, Zs. RI'\{e\} vay, Springer Verlag Budapest, Berlin, 
C. KIM et al., ND2010 Proceedings - Instructions for Authors

Heidelberg 1997 p. 774.

[7] Zs. Rl'\{e\}vay and G.L. Molnl' $\{a\} r$, Radiochimica Acta 91, 361 (2003).

[8] Evaluated Nuclear Structure Data File, a computer file of evaluated experimental nuclear structure data maintained by the National Nuclear Data Center, Brookhaven National Laboratory.

[9] K.J.R. Rosman and P.D.P. Taylor, Pure and Appl. Chem. 70, 217 (1998)

[10] L.V. Groshev, A.M. Demidov, V.N. Lutsenko, and V. Pelekhov,Atlas of the Spectra of Gamma Rays from the Radiative Capture of Thermal Neutrons, Pergamon, London (1961).

[11] R.C. Greenwood and J.H. Reed, Prompt Gamma Rays from Radiative Capture of Thermal Neutrons IIT Research Institute Report IITRI-1193-53 (1965).

[12] N.C. Rasmussen, Y. Hukai, T. inouye, and V.J. Orphan, Thermal Neutron Capture Gamma Ray Spectra of the Elements Massachusetts Institute of Technology Report AFCRL-69-0071 (1969).

[13] V.J. Orphan, N.C. Rasmussen, and T.L. Harper, Line and Continuum Gamma-ray Yields from Thermal Neutron Capture in 75 Elements Gulf General Atomic Report DASA 2570 (GA 10248) (1970).

[14] M.A. Lone, R.A. Leavitt, and D.A. Harrison, At. Data Nucl. Data Tables 28, 511 (1981).

[15] R.C. Reedy and S.C. Frankel At. Data Nucl. Data Tables 80,1 (2002).

[16] R.B. Firestone, H.D. Choi, R.M. Lindstrom, G.L. Molnar, S.F. Mughabghab, R. Paviotti-Corcuera, Zs. Revay, V. Zerkin, and C.M. Zhou, Database of Prompt Gamma Rays from Slow Neutron Capture for Elemental Analysis, IAEA STI/PUB/1263, $251 \mathrm{pp}$ (2007).

[17] N. Bohr, Nature (London) 137, 344 (1936).

[18] C.E. Porter and R.G. Thomas, Phys. Rev. 104, 483 (1956).

[19] P. Axel, Phys. Rev. 126 (1962).

[20] D. Brink (Ph.D. Thesis, Oxford University, 1955).

[21] S. Kadmenskij, V. Markushev, and V. Furman, Sov. J. Nucl. Phys. 37 (1983).

[22] R. Evans, The Atomic Nucleus (McGraw-Hill, New York,1955).

[23] S. Dietrich and B. Berman, At. Data Nucl. Data Tables 38 (1988).

[24] R. Capote et al., Nucl. Data Sheets 110 (2009).

[25] T. von Egidy, H. Schmidt, and A. Bekhami, Nucl. Phys.A 481 (1988).

[26] Jurney, E.T., Bendt, P.J., Browne J.C.: Thermal neutron capture cross section of deuterium. Phys. Rev. C25, 2810 (1982). 


\section{DISCLAIMER}

This document was prepared as an account of work sponsored by the United States Government. While this document is believed to contain correct information, neither the United States Government nor any agency thereof, nor the Regents of the University of California, nor any of their employees, makes any warranty, express or implied, or assumes any legal responsibility for the accuracy, completeness, or usefulness of any information, apparatus, product, or process disclosed, or represents that its use would not infringe privately owned rights. Reference herein to any specific commercial product, process, or service by its trade name, trademark, manufacturer, or otherwise, does not necessarily constitute or imply its endorsement, recommendation, or favoring by the United States Government or any agency thereof, or the Regents of the University of California. The views and opinions of authors expressed herein do not necessarily state or reflect those of the United States Government or any agency thereof or the Regents of the University of California. 